\title{
Domain Texture of the Orthorhombic Phase of $\mathrm{La}_{2-x} \mathrm{Ba}_{x} \mathrm{CuO}_{4}$
}

\author{
Ian Robinson ${ }^{1,2}$ (I) Tadesse A. Assefa $^{1} \cdot$ Yue Cao ${ }^{1,3} \cdot$ Genda Gu $^{1} \cdot$ Ross Harder $^{3} \cdot$ Evan Maxey $^{3} \cdot$ Mark P. M. Dean $^{1}$
}

Received: 19 July 2019 / Accepted: 9 August 2019 / Published online: 23 August 2019

(C) The Author(s) 2019

\begin{abstract}
Bragg coherent diffraction imaging (BCDI) experiments have been carried out at the Advanced Photon Source using a new cryostat system developed to achieve high mechanical stability and low vibrations. We measured the $(012)_{\text {Lто }}$ Bragg peak which is unique to the low-temperature orthorhombic (LTO) phase of micron-sized crystals of the high-temperature superconductor $\mathrm{La}_{2-x} \mathrm{Ba}_{x} \mathrm{CuO}_{4}$ (LBCO) to study the formation of structural domains. Each time the sample was cooled into the orthorhombic phase, the diffraction pattern of domains was different. This confirms the interpretation of pinning of the lower-temperature charge density Wave domains observed in coherent resonant X-ray speckle correlation analysis experiments.
\end{abstract}

Keywords Domain texture $\cdot \mathrm{LBCO} \cdot$ Coherent X-ray diffraction $\cdot$ Return point memory $\cdot$ Pinning $\cdot$ Charge density wave

\section{The Crystal Phases of LBCO}

$\mathrm{La}_{2-x} \mathrm{Ba}_{\mathrm{x}} \mathrm{CuO}_{4}$ (LBCO) is a complex oxide, which was the first high-temperature superconductor (HTS) material, reported by Bednorz and Muller in 1985 [1]. At room temperature, it has a layered high-temperature tetragonal (HTT) structure where the $\mathrm{Cu}-\mathrm{O}$ square layers, which are usually assumed to be the hosts of the superconductivity, form flat planes. The $\mathrm{La} / \mathrm{Ba}$ ions are contained in two spacer layers keeping the $\mathrm{Cu}-\mathrm{O}$ layers about $6 \AA$ apart. Since the $\mathrm{La}^{3+}$ and $\mathrm{Ba}^{2+}$ ions have different valences, a concentration parameter, $x$, determines the density of holes doped into the $\mathrm{Cu}-\mathrm{O}$ layers with respect to the parent compound. At a doping of around $x=0.125$, LBCO forms a charge density wave (CDW) ordering, seen by both neutron and X-ray scattering [2, 3], which competes with the superconductivity, reducing the Meisner effect temperature from around $40 \mathrm{~K}$ to approximately

Ian Robinson

irobinson@bnl.gov

Brookhaven National Laboratory, Upton, NY 11973-5000, USA

2 University College, London WC1E 6BT, UK

3 Argonne National Laboratory, Lemont, IL 60439, USA
$2 \mathrm{~K}$. The CDWs form a domain texture, inferred from the width of its diffraction peak, which gives a measure of the correlation length. Among the cuprates, LBCO has the longest CDW correlation length of $20 \mathrm{~nm}$ [4] discovered so far at zero magnetic field and applied strain. How this CDW state forms is still not fully understood and is the subject of ongoing investigations [5-11].

Upon cooling, LBCO enters a "Low-Temperature Orthorhombic" (LTO) phase below a transition temperature $T_{\mathrm{LTO}}=240 \mathrm{~K}$ (for $x=0.125$ doping). The octahedral coordination shells around the $\mathrm{Cu}$ ions become tilted alternately along the $\mathrm{Cu}-\mathrm{Cu}$ in-plane bond directions to form a doubled unit cell with $a=5.339 \AA, b=5.359 \AA$, and $c=13.24 \AA$. Most cuprates show some form of orthorhombic symmetry breaking, but LBCO has an additional transition to a "LowTemperature Tetragonal" (LTT) phase with a transition temperature $T_{\mathrm{LTT}}=50 \mathrm{~K}$. Here, the octahedral coordination shells switch their rotation axis to follow the in-plane $\mathrm{Cu}-\mathrm{O}$ neighbor directions [12]. Alternate $\mathrm{Cu}-\mathrm{O}$ layers have their tilting in perpendicular directions to maintain an overall tetragonal symmetry with $a=b=5.348 \AA$ and $c=13.24 \AA$. The CDW formation temperature is roughly coincident with $T_{\mathrm{LTT}}$ and it is speculated that the longer range order seen in LBCO is connected somehow with the special feature of LBCO having this additional LTT phase, as this produces rows of oxygen atoms all displaced below the plane, which form potential pinning features for the CDW. 


\section{Domain Pinning Experiments}

Our previous experiments on LBCO $[13,14]$ used soft coherent X-ray diffraction to study the speckle pattern generated by the CDW domains at $10 \mathrm{~K}$. It was found firstly that the speckles were static over long periods [13], hence that the domain texture does not fluctuate, and secondly that the speckle positions were stable in temperature, even as the material was thermally cycled above the $T_{\text {LTT }}$ phase transition where the CDWs melt and the diffraction signal disappears [15]. This result is summarized in Fig. 1, showing the crosscorrelation (defined below) of a region of the speckled diffraction pattern measured at $24 \mathrm{~K}$ before and after cycling up to the designated upper temperature, $T_{\text {cycle, }}$, and back. As can be clearly seen, the speckles remain highly correlated all the way up to $T_{\mathrm{LTO}}=240 \mathrm{~K}$. Only after cycling the temperature above the higher orthorhombic transition temperature, $T_{\mathrm{LTO}}$, did the pattern of speckles, hence domains, rearrange [15].

The important conclusion of that work [15] was that the CDW domains are pinned by the structural domains that form at the LTO phase transition. When the temperature was raised above $T_{\text {LTO }}$ and cooled down again, a different configuration of LTO domains was formed and that led to a different pinning pattern of the CDW domains when the CDW formed again below $T_{\mathrm{LTT}}$. This is a rather surprising result because the CDW domains are very small, $20 \mathrm{~nm}$ across, while the LTO domains are much larger, $700 \mathrm{~nm}$. It was therefore proposed that a nucleation-growth process takes place at the LTO domain walls, newly formed on each temperature cycle, where domains build upon each other progressing out from the domain walls into the LTO domains.

To test this hypothesis, we set out to investigate whether indeed the LTO domains were formed differently on every temperature cycle through $T_{\text {LTO }}$. For this purpose, we employ

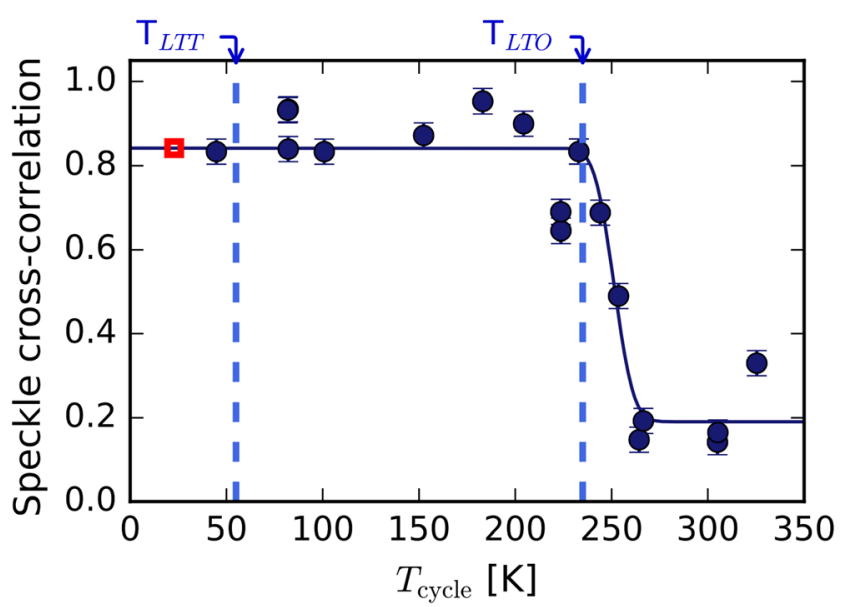

Fig. 1 Cross-correlation of a region of the coherent diffraction pattern of LBCO measured at $(0.24,0,1.5)$ and $24 \mathrm{~K}$ before and after raising the temperature to $T_{\text {cycle }}$. Reproduced from Ref 14 under a CC-BY-4.0 license
Bragg coherent diffraction imaging (BCDI) [16], a powerful three-dimensional (3D) imaging method which is highly sensitive to the locations of the domains in a small crystal and the relative phase shifts from one to the next. It is because of the large momentum transfer, $\mathbf{Q}$, associated with Bragg diffraction peaks that BCDI is extremely sensitive to strain. The relative phase of the diffraction from each small region of the crystal, with displacement $\mathbf{u}$, is given by $\phi=\mathbf{Q} . \mathbf{u}$ [17]. In a domain structure, each domain has a different phase shift, and the coherent interference between all of these gives rise to a rich speckle pattern.

The BCDI method uses a coherent X-ray beam produced by an undulator on a 3rd generation synchrotron. The experiments reported here used the 34-ID-C beamline of the Advanced Photon Source (APS), which has mechanically stable optics and sample positioning, dedicated to BCDI [18]. The experiments used $9 \mathrm{keV}$ X-rays monochromated to $2 \times 10^{-4}$ bandwidth to enhance the longitudinal coherence and a horizontal aperture near the source to provide sufficient spatial coherence to extend over the entire sample [19]. No focusing optics were used.

To obtain images with BCDI, 3D diffraction patterns are recorded by rocking the sample through the Bragg peak and recording the diffraction patterns with fine enough angular steps to resolve the speckles. These patterns are inverted by Fourier transformation using a self-consistent phasing algorithm, where the shape of the crystal is used as a spatial constraint on the extent of the real-space image, called a "support" [20]. In the main part of this work, no images were needed because the 3D speckle distribution is already a unique signature of the domain texture. All data analysis was carried out in the reciprocal space of the measurements instead. The diffraction patterns were oversampled beyond the Shannon limit [21] by using a small crystal and moving the detector far enough away that the speckles could be resolved.

\section{Installation of a Cryostat at 34-ID-C at APS}

Quantum materials are usually interesting at low temperatures and the ordered states of LBCO are all well below room temperature. Cryostats for BCDI are challenging to design because of the extreme sensitivity of the method to vibrations. It is estimated that the cold tip of a typical "Displex" cryostat system that is not optimized for vibrational stability moves by tens of microns every few seconds during the Joule-Thomson cooling cycle. This is incompatible with the $600 \mathrm{~nm}$ focus of the 34-IDC beamline [18] with a small crystal.

For the experiments reported here, we set up and tested a new cryostat from Cryotel corporation. These cryostats use a much higher frequency free-piston Stirling technology cooling system running at a frequency of $60 \mathrm{~Hz}$. It has a miniature design and maintenance-free operation, designed 
to be used on scientific apparatus launched on space missions. The unit fits into the small space available at 34-ID-C and can be positioned by the stepper-motor sample positioning stages. It is too heavy for use with the piezo positioners used for small samples, however. Figure 2 shows a photograph of the installation at 34-ID-C.

We developed a customized sample vacuum chamber with Kapton X-ray windows and electrical feedthroughs for the thermometer connection. This is also seen in Fig. 2. The system worked reasonably well, but the temperature-stabilizing loop of the Cryotel controller was unable to regulate the temperature very well. We understand this might be due to not installing an intermediate-temperature shroud and the lack of a sample heater. Since we only needed rough temperature settings, we used the constant power mode and let it reach a base temperature of $144 \mathrm{~K}$. We then turned off the system to return to room temperature.

\section{Sample Preparation by Grinding}

BCDI needs small crystals to allow their diffraction patterns to be oversampled with respect to the Shannon frequency on the detector [21]; without this constraint, we would not expect to see fringes from the internal domains. The upper size limit for the configuration we used at 34-ID-C is currently around $3 \mu \mathrm{m}$. We have considered several methods for preparing small crystals. Photolithography of a thin film has worked before [22] but is limited to very thin materials, because micron-thick films are rare and several rounds of reactive ion etching would be required to get through micron-thick materials. Focused ion-beam (FIB) methods are also practical for this size range [23], but we were concerned about surface damage effects that might alter the subtle ordering associated with the low-temperature ordering of LBCO.

For this experiment, we decided to grind a larger crystal of LBCO in a pestle. A range of crystal sizes was obtained and these were drop cast in ethanol as a thin film on a silicon wafer substrate. Then the film was coated with $2 \%$ tetraethyl orthosilicate (TEOS) in ethanol and calcined at $450{ }^{\circ} \mathrm{C}$ for $4 \mathrm{~h}$. The TEOS is converted to amorphous $\mathrm{SiO}_{2}$ which forms a covalent bond network between the substrate and the sample. This bonding method has worked well in the past for a number of materials, preventing them from becoming dislodged by the radiation pressure of the X-ray beams [24].

\section{Domain Counting Argument}

Domain textures arise frequently in the complex oxide materials that are important for exploring correlated electron properties. Nanometer-sized domains are common and interference between these gives rise to strongly modulated speckle patterns when they are illuminated with coherent X-ray beams. Inversion of these patterns to real-space images has proven challenging because there appear to be multiple solutions when the standard hybrid input-output (HIO) algorithms [20] are applied, suggesting weak convergence or even nonuniqueness of the resulting image.

Here, we use a simpler counting argument to learn about the number of domains without requiring convergence of a reconstructed image, as illustrated in Fig. 3. A bounded array
Fig. 2 The Cryotel DS2.1 cooling system installed at the sample position of the 34-ID-C beamline at APS

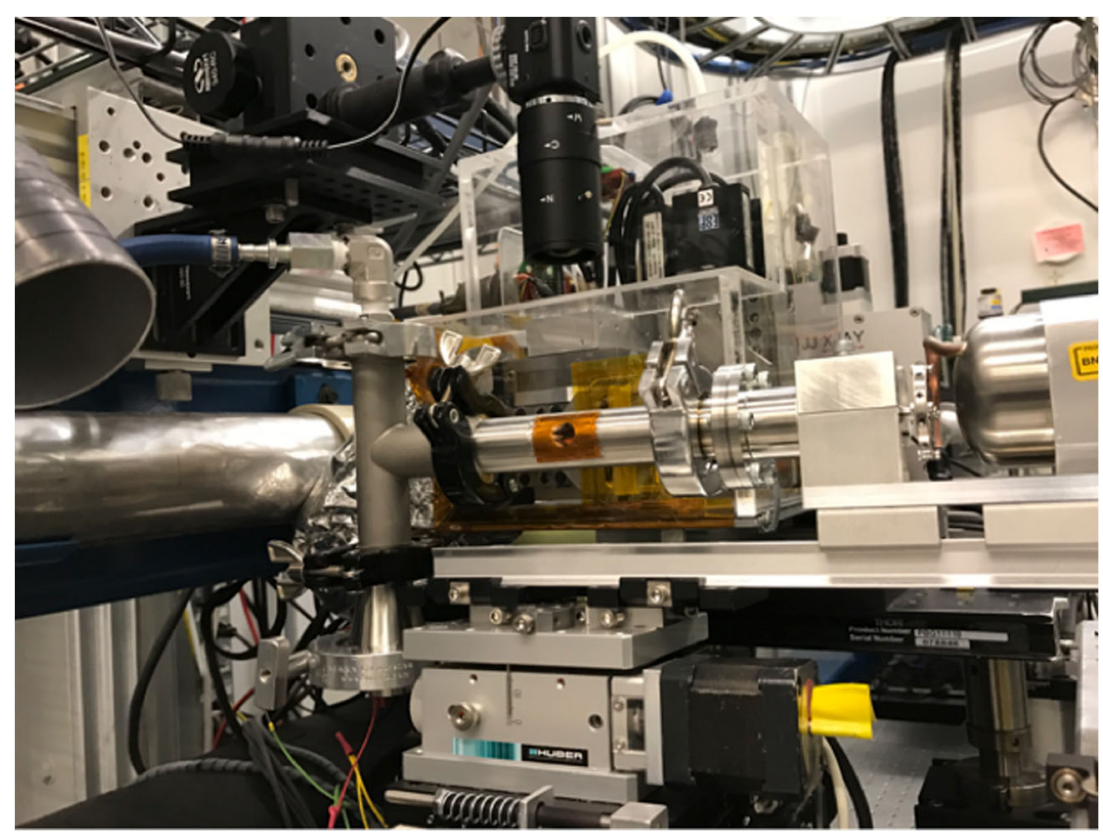




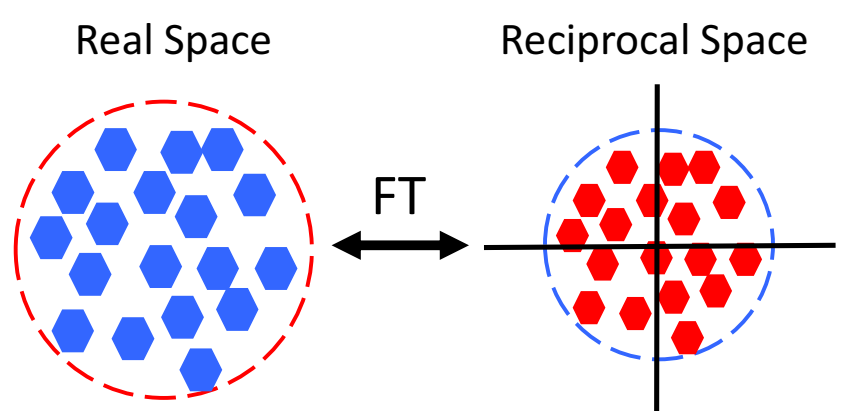

Fig. 3 (a) Sketch of domains within the X-ray illuminated focal spot. (b) Sketch of coherent speckles within a broadened diffraction peak. The two sketches are related by the Fourier transformation (FT)

of small objects, representing domains, is enclosed within a finite region, representing the extent of the X-ray beam (or a finite-sized sample, whichever is smaller) in Fig. 3(a). Its diffraction pattern, obtained by Fourier transformation (FT), is shown schematically in Fig. 3(b), with speckles enclosed within a diffraction peak envelope; without X-ray coherence, this peak would have a smooth broad shape of width $2 \pi / w$, where $w$ is the typical domain size in real space. When the beam becomes coherent, the envelope width of the speckle cluster is the same, $2 \pi / w$. Meanwhile, the width of each speckle is $2 \pi / b$, where $b$ is the beam size in real space.

In one dimension, the number of domains in the beam is $w / b$, while the number of speckles in the envelope is $(2 \pi / b) /(2 \pi / w)=$ $w / b$, which is the same. In 2 or 3 dimensions, the same argument is true in each direction of space. So long as the domains mostly fill the space, this is a general result that the total number of speckles in reciprocal space is numerically equal to the total number of coherently illuminated domains within the ensemble in real space, whether evaluated in $1 \mathrm{D}, 2 \mathrm{D}$, or 3D. This argument assumes the domains are space-filling and touch each other.

\section{Diffraction Patterns upon Cycling Temperature}

As the (012) $)_{\text {LTO }}$ diffraction peak is a thousand times weaker than the HTT peaks, we looked for an LBCO crystal in the micrometer size range, as discussed previously. Therefore, and to avoid further losses due to the minor vibration of the cryostat, we used unfocused X-rays with an entrance slit size of $100 \times 50$ microns. As the sample is substantially smaller than the X-ray size and the sample stays within the footprint of the flat wavefront, the micron-size LBCO crystal is effectively illuminated by coherent X-ray in this non-standard setup.

After selecting an appropriately sized crystal by its (012) Lто diffraction peak and centering the crystal within the entrance slits, a full rocking curve BCDI scan was collected at $144 \mathrm{~K}$. Three hundred angular steps of $0.001^{\circ}$ were made with $10 \mathrm{~s}$ exposures on a $p=55 \mu \mathrm{m}$ pixel Medipix area detector placed $D=2.2 \mathrm{~m}$ from the sample. The temperature was then raised over $30 \mathrm{~min}$ to $300 \mathrm{~K}$ and it was verified that the diffraction intensity at the peak center had vanished around $240 \mathrm{~K}$ within the precision of the thermometer readback. The sample was then cooled again to $144 \mathrm{~K}$ over $30 \mathrm{~min}$ and the peak was seen to return. The peak was found to be still wellcentered in the $100 \times 50$-micron beam-defining slits, so it was very unlikely to have returned to find a different crystal. The sample density was such that it had taken several hours of searching angle and position to find the crystal in the first place. A second full BCDI rocking scan was then collected at $144 \mathrm{~K}$. Three more temperature cycles following the same procedure were carried out and the crystal returned each time. This resulted in 5 full diffraction patterns all measured on the same crystal at $144 \mathrm{~K}$ with a temperature excursion well above $T_{\mathrm{LTO}}=240 \mathrm{~K}$ in between each one.

The central frame of each of the five diffraction patterns is shown in Fig. 4. A clear pattern of speckles is seen with good contrast in each case. While the overall shape of the peak is the same, the distribution of speckles within it is different. For the third temperature cycle, the scan was repeated and the second measurement of the central slice is clearly seen to be similar. The immediate conclusion drawn from the simple observation that these data differ from each other is that the LTO domain structure is indeed rearranged by cycling through $T_{\mathrm{LTO}}$, as predicted by the CDW pinning experiments described above.

The dimensions of the speckle pattern allow estimates to be made about the LTO domains. To reach the (012) LTO $_{\text {p }}$ peak, the detector was positioned with a compound angle of $15.2^{\circ}$ in the horizontal plane (delta) and $11.4^{\circ}$ in the vertical plane (gamma) to make a total Bragg angle of $18.9^{\circ}$ (2-theta) which corresponds to the spacing of the 012 planes at $9 \mathrm{keV}(\lambda=$
$\mathrm{P} 1$
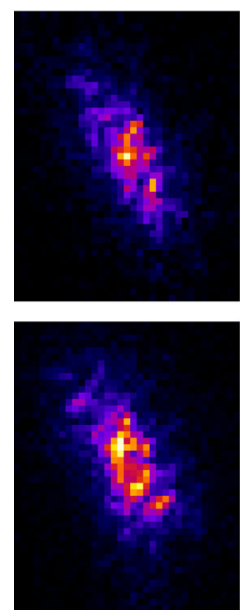

P5
P2
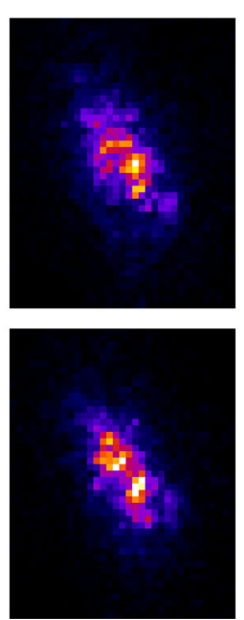

P4
P3A

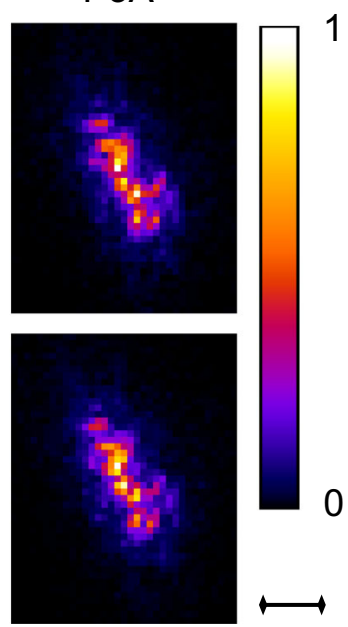

P3B $\quad 0.001 \AA^{-1}$
Fig. 4 Coherent diffraction patterns of a small crystal of LBCO at the $(012)_{\text {LтO }}$ Bragg reflection at $144 \mathrm{~K}$. The pixel size is $55 \mu \mathrm{m}$ on an area detector placed $2.2 \mathrm{~m}$ from the sample. The labels are explained in the text 
$1.38 \AA$ ). The direction of the peak broadening in Fig. 4 is transverse, around the (012) LTO powder ring; most of the speckle structure follows that direction, too. Each speckle is roughly circular with a spacing of approximately $s=4$ pixels. From the speckle shape and size, the shape of the crystal can be estimated to be roughly spherical with a diameter of $\lambda D / \mathrm{sp}=$ $1.4 \mu \mathrm{m}$. The 3D shape of the diffraction peak is seen in the intensity distribution along the rocking scan.

As can be seen in all diffraction patterns in Fig. 4, there are typically five speckles in the transverse direction and two in the radial direction. Roughly five speckles are seen in the other transverse direction along the rocking curve (data not shown). This oblate ellipsoidal shape of the Bragg peak informs us about the shape of the domains. The method described in the "Domain Counting Argument" section above, informs us that, within the 1.4- $\mu \mathrm{m}$ sphere, there are roughly five domains across the transverse direction and two along the (012) radial direction. Therefore, the LTO domains, roughly $280 \mathrm{~nm}$ wide and $700 \mathrm{~nm}$ long, correspond well to the estimates made from the incoherent envelope of the 3D diffraction pattern, as used for the estimates made in our previous publication [15]. An a-b anisotropy within the orthorhombic phase was observed before in $c$-axis darkfield transmission electron microscope (TEM) images, with roughly the same dimensions $[25,26]$. In both dark-field TEM and BCDI, domain walls are only visible when there is some component of phase shift along the momentum transfer direction; this can also lead to an apparent anisotropy, as some domain walls are invisible.

To verify at a quantitative level that the five 3D diffraction patterns are different, we calculate the 3D sample Pearson cross-correlation coefficient between the intensity values of pairs of them, $A_{i}$ and $B_{i}$,

$$
\mathrm{r}=\frac{\sum\left(\mathrm{A}_{\mathrm{i}}-\overline{\mathrm{A}}\right)\left(\mathrm{B}_{\mathrm{i}}-\overline{\mathrm{B}}\right)}{\sqrt{\sum\left(\mathrm{A}_{\mathrm{i}}-\overline{\mathrm{A}}\right)^{2} \sum\left(\mathrm{B}_{\mathrm{i}}-\overline{\mathrm{B}}\right)^{2}}}
$$

The summations are carried out over the central volumes of the peaks, $A_{i}$ and $B_{i}$, containing $11 \times 11 \times 41$ pixels in the $x, y$, and $z$ directions respectively, with $x$ and $y$ being the detector directions and $z$ being the angular theta-scan direction. $\overline{\mathrm{A}}$ and $\overline{\mathrm{B}}$ represent the average intensity values. To compensate for any change of alignment during the temperature cycling, the $3 \mathrm{D}$ diffraction patterns were shifted in all directions until the largest correlation was found. The patterns $\mathrm{P} 3 \mathrm{~A}$ and $\mathrm{P} 3 \mathrm{~B}$, where the measurement was repeated without temperature cycling, gave $r=0.88$, which is an indication of how much the vibration effects and photon statistics contribute to the measurement. The correlation values obtained are given in Table 1 .

We conclude from these tests that the 3D diffraction patterns change significantly in the detailed configuration of their speckles upon temperature cycling above $T_{\mathrm{LTO}}=240 \mathrm{~K}$. The
Table 1 Values of the correlation coefficient, $r$, between the 3D diffraction patterns measured after each temperature cycle. The reason the table is not exactly symmetric is that a fixed volume was selected for the column pattern and the row pattern was shifted for optimum alignment with respect to it, so the final volumes are not quite the same

\begin{tabular}{llllll}
\hline & P1 & P2 & P3A & P4 & P5 \\
\hline P1 & 1 & 0.541 & 0.572 & 0.543 & 0.549 \\
P2 & 0.495 & 1 & 0.478 & 0.564 & 0.543 \\
P3A & 0.474 & 0.516 & 1 & 0.535 & 0.604 \\
P4 & 0.507 & 0.562 & 0.538 & 1 & 0.570 \\
P5 & 0.541 & 0.586 & 0.603 & 0.596 & 1 \\
\hline
\end{tabular}

lower level of correlations, around 0.5 , is due to different patterns of intensity modulation in each pattern, departing from the mean distribution expected for incoherent diffraction. The degree of coherence of the X-ray beam used in these experiments, which determines the amount of intensity modulation or "fringe visibility," is expected to be quite high and contributes to the drop of correlations seen. This confirms that the detailed configuration of domains is different in every case. There is little indication in Table 1 that any of the patterns is more similar than the others, with the highest correlation, $r=0.60$, being between patterns $\mathrm{P} 5$ and $\mathrm{P} 3 \mathrm{~A}$, which were two temperature cycles apart. So it is fairly safe to conclude that there are many possible domain configurations available on cooling the sample through the LTO phase transition.

\section{Images, Limitations, and Conclusions}

The results presented here confirm that the LTO domain texture is rearranged upon heating LBCO through its LTO phase transition at $T_{\mathrm{LTO}}=240 \mathrm{~K}$. This adds strong support to the arguments made in Ref [15] to explain the high-temperature memory of the CDW domain texture and its abrupt loss upon crossing $T_{\mathrm{LTO}}$.

These conclusions were drawn directly in reciprocal-space speckle patterns obtained by coherent X-ray diffraction from small crystals of LBCO. While the results are unambiguous in reciprocal space, there is a strong interest to view the domains in real-space images. So we reconstructed a real-space image from the $3 \mathrm{D}$ diffraction dataset $\mathrm{P} 3 \mathrm{~A}$ discussed above in the middle of the temperature cycle series. We used the $\mathrm{HIO}$ algorithm in alternation with simple error reduction, starting from a support of half the size of the real-space array size, corresponding to two pixels per fringe [27]. This gave a least-squares error of $\chi^{2}=$ 0.033 between the data amplitude and the values calculated by Fourier transforming the image iterate. After 200 iterations, we started using "Shrinkwrap" [28] to reduce the support to the shape of the particle with a threshold value of 0.15 . This increased the chi square to 0.132 but produced a clean image shown in Fig. 5 . The 


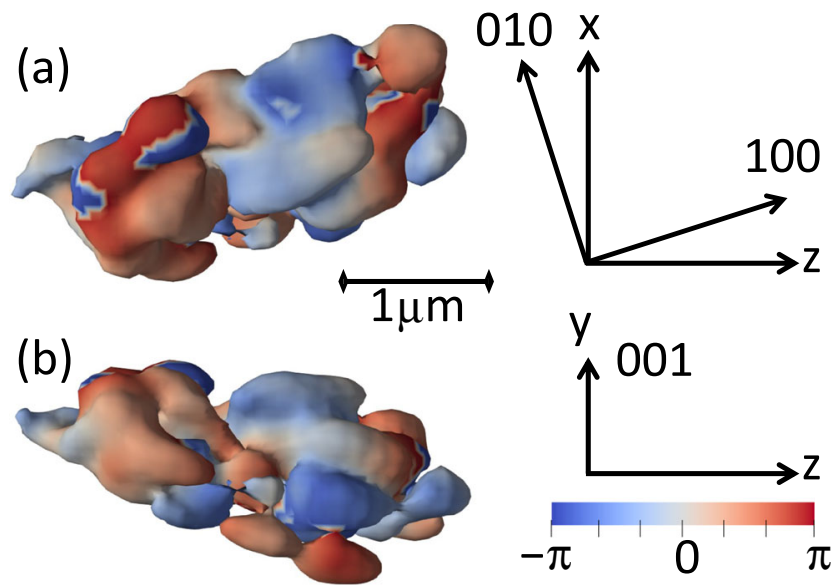

Fig. 5 Two views of the Bragg coherent diffraction image of the LBCO crystal examined in this study, imaged using the (012) orthorhombic peak. An isosurface contour of the image amplitude is shown at $15 \%$ of the maximum value. The isosurface is colored according to the phase value at that contour level to indicate the relative phases of the different domains

domains can be clearly seen in an isosurface contour-map image viewed using "Paraview" [29].

The axes used to display the image in Fig. 5 are the "laboratory" coordinate frame, obtained by a linear transformation from the measurement coordinates, the stack of 2D diffraction patterns (like those shown in Fig. 4) for each angle on the rocking curve [16]. The $x$-axis is horizontal and the $y$-axis vertical, perpendicular to the incoming beam, while $z$ is along the beam direction. Since the crystal's orientation matrix was fully aligned by its (012) LTO and (113) $)_{\text {LTO }}$ Bragg reflections, we know its crystallographic axis directions, which are labeled on Fig. 5 using the LTO coordinate system. It is clear that both the shape of the crystal and the boundaries of the domains within it follow the $[100]_{\text {LTO }}$ and $[010]_{\text {LTO }}$ crystal directions. These are both $\{110\}_{\text {HTT }}$ directions of the HTT high-temperature phase. This crystal shape could arise due to preferential cleavage planes during the grinding process. It is interesting that the domain boundaries appear to follow the shape of the micro-crystal rather than the $\{100\}_{\text {HTT }}$ directions seen in TEM studies of bulk crystals [25, 26].

The dimensions of the crystal are $2.3 \mu \mathrm{m}$ along [100], $1.1 \mu \mathrm{m}$ along [010], and $1.1 \mu \mathrm{m}$ along [001]. This corresponds roughly to the size estimated above from the speckle size. The domain sizes also correspond to the width of the diffraction peak envelope. The discussion in the "Diffraction Patterns upon Cycling Temperature" section above was based on single detector images, which correspond roughly to the $x-y$ plane of the laboratory coordinate frame. It can be seen in Fig. 5 that there are indeed about five rows of domains within the crystal along $y$ and about two in $x$, as determined from the domain counting argument above applied to the number of speckles in Fig. 4. There are also about five domains along the crystal in the $z$-direction, corresponding to the number of speckles in the rocking curve direction (data not shown).

The resolution of the image is about $220 \mathrm{~nm}$ based on line cuts in the three axis directions. This leads to significant rounding of the shapes of the domains and to apparent gaps between them, depending on the contour level chosen. The phases of the image are different on the surface of each domain and are mostly flat across the domain. More detailed information could be obtained by looking at the $3 \mathrm{D}$ phase distribution inside the domains, but it is likely to be similar to that shown on the surface. The phase differences between neighboring domains, arising from the domains wall structures, correspond to sub-unit-cell lattice displacements of one domain with respect to the others; these are responsible for the interference needed to give rise to speckled diffraction patterns. A few domains can be seen to have phase gradients on their surfaces; these correspond to small rotations of the domain within the crystal lattice, which gives rise to phase ramps [30].

The image in Fig. 5 is satisfying because it agrees with the information obtained by quantitative analysis of the coherent X-ray diffraction data given in the first part of this paper. The images of phase domain structures are however found to be somewhat unreliable in general. This one was fairly reproducible as a function of shrinkwrap threshold, zero-padding of the arrays, and background cut-off level. It did vary slightly with the choice of random numbers used to seed the phasing algorithm. This is a general problem of BCDI, which was developed for determining the shapes of nanocrystals $[16,17]$, for which it works very well. Small strains in well-shaped nanoparticles can be reliably imaged too, but when the phase structure becomes strong, the reproducibility of the solution becomes worse. It has been known for a long time that the solution of the phase problem with oversampled diffraction data is only an overdetermined mathematical problem by virtue of the real-space constraint imposed by the support. Even then, the phase problem is prone to multiple solutions in dimensions less than two [31]. The support is apparently a more effective constraint for the nanoparticle inversion problem than the phase domain problem. The application of shrinkwrap to the support also resolves the "propagation uniqueness" issues [32], where the focal plane of the image is the one with the smallest support. This is also more effective in the nanoparticle case than the phase domain inversion problem.

Funding Information Work at Brookhaven National Laboratory was supported by the U.S. Department of Energy (DOE), Office of Science, Office of Basic Energy Sciences, under Contract No. DE-SC0012704. 
The beamline 34- ID-C was built with U.S. National Science Foundation grant DMR-9724294. This research used resources of the Advanced Photon Source, a DOE Office of Science User Facility. All work at Argonne National Laboratory was supported the DOE Office of Science by under Contract No. DE-AC02-06CH11357.

Open Access This article is distributed under the terms of the Creative Commons Attribution 4.0 International License (http:// creativecommons.org/licenses/by/4.0/), which permits unrestricted use, distribution, and reproduction in any medium, provided you give appropriate credit to the original author(s) and the source, provide a link to the Creative Commons license, and indicate if changes were made.

\section{References}

1. Bednorz, J.G., Müller, K.A.: Possible high Tc superconductivity in the Ba-La-Cu-O system. Zeitschrift für Physik B Condensed Matter. 64, 189-193 (1986)

2. Tranquada, J.M., Sternlieb, B.J., Axe, J.D., Nakamura, Y., Uchida, S.: Evidence for stripe correlations of spins and holes in copper oxide superconductors. Nature. 375, 561 (1995)

3. Fujita, M., Goka, H., Yamada, K., Tranquada, J.M., Regnault, L.P.: Stripe order, depinning, and fluctuations in $\mathrm{La}_{1.875} \mathrm{Ba}_{0.125} \mathrm{CuO}_{4}$ and $\mathrm{La}_{1.875} \mathrm{Ba}_{0.075} \mathrm{Sr}_{0.050} \mathrm{CuO}_{4}$. Phys. Rev. B. 70, 104517 (2004)

4. Thampy, V., Dean, M.P.M., Christensen, N.B., Steinke, L., Islam, Z., Oda, M., Ido, M., Momono, N., Wilkins, S.B., Hill, J.P.: Rotated stripe order and its competition with superconductivity in $\mathrm{La}_{1.88} \mathrm{Sr}_{0.12} \mathrm{CuO}_{4}$. Phys. Rev. B. 90, 100510 (2014)

5. Först, M., Tobey, R.I., Bromberger, H., Wilkins, S.B., Khanna, V., Caviglia, A.D., Chuang, Y.-D., Lee, W.S., Schlotter, W.F., Turner, J.J., Minitti, M.P., Krupin, O., Xu, Z.J., Wen, J.S., Gu, G.D., Dhesi, S.S., Cavalleri, A., Hill, J.P.: Melting of charge stripes in vibrationally driven $\mathrm{La}_{1.875} \mathrm{Ba}_{0.125} \mathrm{CuO}_{4}$ : assessing the respective roles of electronic and lattice order in frustrated superconductors. Phys. Rev. Lett. 112, 157002 (2014)

6. Fausti, D., Tobey, R.I., Dean, N., Kaiser, S., Dienst, A., Hoffmann, M.C., Pyon, S., Takayama, T., Takagi, H., Cavalleri, A.: Lightinduced superconductivity in a stripe-ordered cuprate. Science. 331, 189-191 (2011)

7. Nicoletti, D., Casandruc, E., Laplace, Y., Khanna, V., Hunt, C.R., Kaiser, S., Dhesi, S.S., Gu, G.D., Hill, J.P., Cavalleri, A.: Opticallyinduced superconductivity in striped $\mathrm{La}_{2-\mathrm{x}} \mathrm{Ba}_{\mathrm{x}} \mathrm{CuO}_{4}$ by polarization-selective excitation in the near infrared. Phys. Rev. B. 90, 100503 (2014)

8. Miao, H., Lorenzana, J., Seibold, G., Peng, Y.Y., Amorese, A., YakhouHarris, F., Kummer, K., Brookes, N.B., Konik, R.M., Thampy, V., Gu, G.D., Ghiringhelli, G., Braicovich, L., Dean, M.P.M.: Hightemperature charge density wave correlations in $\mathrm{La}_{1.875} \mathrm{Ba}_{0.125} \mathrm{CuO}_{4}$ without spin-charge locking. Proc. Natl. Acad. Sci. U.S.A. 114, 12430 (2017)

9. Miao, H., Ishikawa, D., Heid, R., Le Tacon, M., Fabbris, G., Meyers, D., Gu, G.D., Baron, A.Q.R., Dean, M.P.M.: Incommensurate phonon anomaly and the nature of charge density waves in cuprates. Phys. Rev. X. 8, 011008 (2018)

10. H. Miao, R. Fumagalli, M. Rossi, J. Lorenzana, G. Seibold, F. Yakhou-Harris K. Kummer, N. B. Brookes, G. D. Gu, L. Braicovich, G. Ghiringhelli, M. P. M. Dean Formation of incommensurate charge density waves in cuprates arXiv: 1906.07149 (2019)

11. Keimer, B., Kivelson, S.A., Norman, M.R., Uchida, S., Zaanen, J.: From quantum matter to high-temperature superconductivity in copper oxides. Nature. 518, 179 (2015)

12. Bozin, E.S., Zhong, R., Knox, K.R., Gu, G., Hill, J.P., Tranquada, J.M., Billinge, S.J.L.: Reconciliation of local and long-range tilt correlations in underdoped $\mathrm{La}_{2-\mathrm{x}} \mathrm{Ba}_{\mathrm{x}} \mathrm{CuO}_{4}(0 \leq \mathrm{x} \leq 0.155)$. Phys. Rev. B. 91, 054521 (2015)

13. Chen, X.M., Thampy, V., Mazzoli, C., Barbour, A.M., Miao, H., Gu, G.D., Cao, Y., Tranquada, J.M., Dean, M.P.M., Wilkins, S.B.: Remarkable stability of charge density wave order in $\mathrm{La}_{1.875} \mathrm{Ba}_{0.125} \mathrm{CuO}_{4}$. Phys. Rev. Letts. 117, 167001 (2016)

14. Thampy, V., Blanco-Canosa, S., Garcia-Fernandez, M., Dean, M.P.M., Gu, G.D., Först, M., Loew, T., Keimer, B., Le Tacon, M., Wilkins, S.B., Hill, J.P.: Comparison of charge modulations in $\mathrm{La}_{1.875} \mathrm{Ba}_{0.125} \mathrm{CuO}_{4}$ and $\mathrm{YBa}_{2} \mathrm{Cu}_{3} \mathrm{O}_{6.6-\mathrm{d}}$. Phys. Rev. B. 88, 024505 (2013)

15. Chen, X.M., Cao, Y., Mazzoli, C., Thampy, V., Barbour, A.M., Hu, W., Lu, M., Assefa, T., Miao, H., Fabbris, G., Gu, G.D., Tranquada, J.M., Dean, M.P.M., Wilkins, S.B., Robinson, I.K.: Charge density wave memory in a cuprate superconductor. Nature Comms. 10(1435), 1-6 (2019)

16. Pfeifer, M.A., Williams, G.J., Vartanyants, I.A., Harder, R., Robinson, I.K.: Three-dimensional mapping of a deformation field inside a nanocrystal. Nature. 442, 63-66 (2006)

17. Robinson, I., Harder, R.: Coherent diffraction imaging of strains on the nanoscale. Nat. Mater. 8, 291-298 (2009)

18. Li, L., Li, X., Xie, Y.Y., Maxey, E., Harder, R.: Methods for operando coherent X-ray diffraction of battery materials at the Advanced Photon Source. J. Synch. Rad. 26, 220-229 (2019)

19. Ian, K.: Robinson, optimisation of coherent X-ray diffraction imaging at ultrabright synchrotron sources. Zeitschrift fur Kristallographie Suppl. 27, 27-35 (2008)

20. Fienup, J.R., Crimmins, T.R., Holsztynski, W.: Reconstruction of the support of an object from the support of its autocorrelation. J. Opt. Soc. Am. 72, 610-624 (1982)

21. Shannon, C.E.: Communication in the presence of noise. Proc. Inst. Radio Eng. 37, 10-21 (1949)

22. Xiong, G., Moutanabbir, O., Huang, X., Paknejad, S.A., Shi, X., Harder, R., Reiche, M., Robinson, I.K.: In-plane elastic relaxation in single ultrathin strained silicon-on-insulator structure. Appl. Phys. Letts. 99(114103), 114103 (2011)

23. Hofmann, F., Tarleton, E., Harder, R., Phillips, N., Ma, P.-W., Clark, J., Robinson, I., Abbey, B., Liu, W., Beck, C.: 3D lattice distortions and defect structures in ion-implanted nano-crystals. Nat. Sci. Rep. 7(45993), (2017)

24. Monteforte, M., Estandarte, A., Chen, B., Harder, R., Huang, M., Robinson, I.: Novel silica stabilisation method for the analysis of fine nanocrystals using coherent X-ray diffraction imaging. J. Synch. Rad. 23, 953 (2016)

25. Zhu, Y., Moodenbaugh, A.R., Cai, Z.X., Tafto, J., M.: Suenaga, and D. O. Welch, Tetragonal-orthorhombic structural modulation at low temperature in $\mathrm{La}_{2-x} \mathrm{Ba}_{\mathrm{x}} \mathrm{CuO}_{4}$. Phys. Rev. Lett. 73, 3026-3029 (1994)

26. Horibe, Y., Inoue, Y., Koyama, Y.: Direct observation of dynamic local structure in $\mathrm{La}_{2-\mathrm{x}} \mathrm{Sr}_{\mathrm{x}} \mathrm{CuO}_{4}$ around $\mathrm{x}=0.12$. Phys. Rev. B. 61, 11922 (2000)

27. Clark, J.N., Beitra, L., Xiong, G., Higginbotham, A., Fritz, D.M., Lemke, H.T., Zhu, D., Chollet, M., Williams, G.J., Messerschmidt, M., Abbey, B., Harder, R.J., Korsunsky, A.M., Wark, J.S., Robinson, I.K.: Ultrafast three dimensional imaging of lattice dynamics in gold nanocrystals. Science. 341, 56 (2013)

28. Marchesini, S., He, H., Chapman, H.N., Hau-Riege, S.P., Noy, A., Howells, M.R., Weierstall, U., Spence, J.C.H.: X-ray image reconstruction from a diffraction pattern alone. Phys. Rev. B. 68, $140101(2003)$

29. Ahrens, J., Geveci, B., Law, C.: ParaView: an end-user tool for large data visualization. Elsevier, Visualization Handbook (2005)

30. Aranda, M.A.G., Berenguer, F., Bean, R.J., Shi, X., Xiong, G., Collins, S.P., Nave, C., Robinson, I.K.: Coherent X-ray diffraction characterization of twinned microcrystals. J. Synch. Rad. 17, 751760 (2010) 
31. Bates, R.H.T.: Fourier phase problems are uniquely solvable in more than one dimension I: Underlying theory. Optik. 61, 247$262(1982)$

32. Huang, X., Harder, R., Xiong, G., Shi, X., Robinson, I.: Propagation uniqueness in coherent diffractive imaging. Phys. Rev. B. 83(224109), (2011)
Publisher's Note Springer Nature remains neutral with regard to jurisdictional claims in published maps and institutional affiliations. 